CAHIERS DE

NARRATOLOGIE

\section{Cahiers de Narratologie}

Analyse et théorie narratives

$36 \mid 2019$

Rhétorique et représentations de la culture mafieuse. Images, rituels, mythes et symboles

\title{
L'imaginaire de la Google generation criminelle : les profils Facebook des jeunes de la Camorra
}

\section{Marcello Ravveduto}

Translator. Manuela Bertone (de l'italien)

\section{(2) OpenEdition}

\section{Journals}

Electronic version

URL: http://journals.openedition.org/narratologie/9942

ISSN: 1765-307X

\section{Publisher}

LIRCES

Electronic reference

Marcello Ravveduto, «L'imaginaire de la Google generation criminelle : les profils Facebook des jeunes de la Camorra », Cahiers de Narratologie [Online], 36 | 2019, Online since 20 December 2019, connection on 20 December 2019. URL : http://journals.openedition.org/narratologie/9942

This text was automatically generated on 20 December 2019.

Article L.111-1 du Code de la propriété intellectuelle. 


\title{
L'imaginaire de la Google generation criminelle : les profils Facebook des jeunes de la Camorra
}

\author{
Marcello Ravveduto
}

Translation : Manuela Bertone (de l'italien)

1 Les camorristi utilisent-ils les réseaux sociaux ? Et si oui, comment ? Pour répondre à ces deux questions, il faut considérer deux aspects préliminaires. D'abord, un aspect méthodologique. Serge Gruzinski propose de réagir à la nature fugace du contemporain en développant «une compréhension et une mise en forme du présent à travers les sens, mais d'un présent avec des touches de passé et des pointes de futur » ${ }^{1}$. Et il donne comme exemple une photo de l'arc romain de Tazoult, en Algérie. L'image de l'artefact, devenu la cage d'un terrain de football improvisé, restitue la profondeur du passé en l'éloignant du caractère fragmentaire du présent : « Réduit depuis longtemps à l'état de ruine, l'arc remplace la cage de but, sans doute trop onéreuse ou trop compliquée à installer. Relique oubliée d'un passé révolu, il a donc été recyclé dans un sport devenu l'un des fleurons sportifs les plus spectaculaires et les plus rentables de la mondialisation $»^{2}$. L'arc est une sorte de portail multidimensionnel "puisqu'il fait le lien entre la mondialisation contemporaine et les temps d'une romanité triomphante, lointaine ébauche de ce qui se met en place aujourd'hui $»^{3}$. C'est un symbole qui évoque une série ininterrompue d'invasions, de conquêtes et de révoltes d'une Afrique passée par la colonisation et la décolonisation au cours de sa longue histoire :

Le ballon de football qui s'écrase sur la pierre traverse de multiples mémoires qui résonnent de siècle en siècle. Locale et nationale, antique et contemporaine, coloniale et impériale, africaine et méditerranéenne, païenne, chrétienne et musulmane, la trame sur laquelle vient se greffer cette petite scène "sans histoire" est infiniment plus riche que la banalité du sujet ne le laissait augurer"

Gruzinski nous invite à réfléchir sur la possibilité d'interpréter le présent «avec les fragments [du passé] que le temps [...] a préservés», afin d'identifier et de contextualiser les différentes « couches » qui constituent un instant ou une scène ; pour retracer « les espaces et les temps qui convergent en un même lieu, décrypter le hors- 
champ, s'ouvrir aux réminiscences qu'inspire l'image $»^{5}$. Il s'agit de savoir " invoquer » le présent avec un "regard historique », ou, si l'on veut, avec le regard de l'historien capable de relier des connaissances lointaines et proches « en jouant sur des échelles multiples $»^{6}$. Dans cette perspective, la sphère du numérique devient une unité prismatique dans laquelle les images du passé sont réfléchies, décomposées et recomposées.

3 Le deuxième aspect est de nature structurelle. Le rapport Digital 2018 a montré que le nombre d'utilisateurs connectés à Internet dans le monde dépasse les 4 milliards; plus de la moitié de la population mondiale est en ligne. L'utilisation des médias sociaux croît en même temps que les utilisateurs connectés, en hausse de $13 \%$ par rapport à 2017 : il y a plus de 3 milliards d'utilisateurs actifs dans le monde, 9 sur 10 via des appareils mobiles. "En Italie, $73 \%$ de la population est connectée (43 millions de personnes) [...]. Au cours de l'année 2017, il y a eu une augmentation de 4 millions de personnes connectées à Internet ( $+10 \%$ par rapport à l'année précédente) et une augmentation de 3 millions d'utilisateurs de médias sociaux (+10\% par rapport à l'année précédente) $\aleph^{7}$. Les Italiens passent sur Internet environ 6 heures par jour (presque deux fois plus longtemps que devant la télévision): pendant 2 de ces 6 heures ils utilisent une plateforme de médias sociaux. La part du lion revient à Facebook qui, avec plus de 34 millions d'utilisateurs, fédère $57 \%$ de la population italienne ${ }^{8}$. Ainsi, soit les mafieux font partie des $43 \%$ restants soit ils sont intégrés dans cette majorité s'élevant à 57\%. Comme l'a écrit Tonino Cantelmi :

on parle de révolution numérique parce que la technologie est devenue un environnement à habiter, une extension de l'esprit humain, un monde entrelacé avec le monde réel et qui détermine de véritables restructurations cognitives, émotionnelles et sociales de l'expérience, un monde pouvant redéfinir la construction de l'identité et des relations, ainsi que le vécu des individus9.

Par conséquent, si le virtuel est un prolongement de la vie réelle, l'expérience criminelle fait aussi partie intégrante de l'écosystème numérique. Le loubard, le délinquant, le mafieux cherchent dans les social networks un horizon culturel, une Weltanschauung criminelle à partager par le biais de contenus multimédia.

5 Le passage de la civilisation industrielle à la civilisation informatique est en train de restructurer les processus cognitifs : l'alphabétisation numérique se fait par induction et auto-apprentissage. Ceux qui se servent de Google, Facebook, YouTube ou de tout autre outil sur le Net utilisent leur intuition et leur capacité d'émulation, c'est-à-dire des compétences et des talents indépendants du niveau scolaire individuel. Même ceux qui n'ont pas atteint un niveau suffisant d'éducation analogique peuvent se sentir à l'aise dans l'expérience virtuelle; ils peuvent faire entendre leur voix et prendre la parole sans avoir à passer par les canaux de la scolarisation, du mérite, des connaissances, du professionnalisme ou des compétences techniques et scientifiques. Ce qui compte, ce n'est pas le "savoir ", mais le "savoir-faire » ou " méta-technologie " (Wright 2000), une pratique qui permet l'assimilation de l'usage individuel et social d'une nouvelle technologie, et qui marque une rupture avec le passé : le transfert de technologie, en effet, est le partage dans le présent d'une innovation qui devient habituelle dans un contexte relationnel. La métatechnologie fait partie de la formation empirique des nouvelles générations. La diffusion de la téléphonie mobile, par exemple, a transformé la communication par l'utilisation massive de messages de texte. 
D'abord les SMS, puis les applications de messagerie instantanée (App) ont transformé le langage en une sorte d'«oralité écrite» (Minnini 2010) dont la structure est asymptotique et souvent agrammatique: "les SMS comprennent des formes linguistiques particulières qui visent à compenser l'absence de codes communicatifs, gestuels, expressifs et proxémiques $»^{10}$. Ceux qui ne participent pas au « jeu » risquent de tomber dans l'abîme de la fracture numérique, où la technologie perd son sens et devient un problème, plutôt qu'une opportunité. Même si, comme l'explique le psychologue Giuseppe Riva, ce n'est pas la date de naissance qui identifie les «digital natives $»^{11}$ mais leur capacité à utiliser la technologie de manière intuitive, il n'en reste pas moins que ce sont principalement les générations des personnes nées entre le milieu des années 70 et le début des années 2000 qui ont diffusé mondialement, grâce à l'utilisation de smartphones, le partage de contenus publics et privés sur les réseaux sociaux :

La facilité d'utilisation de ces appareils a éliminé la barrière de la langue qui a longtemps été la principale exigence de base pour accéder au potentiel des nouveaux médias. Aujourd'hui, il n'est plus nécessaire de savoir lire ou écrire pour pouvoir utiliser les smartphones et les tablettes, il suffit de savoir contrôler ses doigts $^{12}$

7 L'apparition simultanée des mobile devices et des réseaux sociaux (2007) a décrété la naissance d'un nouvel espace social qui a été défini «l'inter-réalité », un espace dans lequel les réseaux numériques en ligne et les réseaux sociaux hors ligne fusionnent: «Ce qui caractérise l'inter-réalité est l'échange entre les différentes dimensions: le monde numérique influence le réel et vice versa; la dimension publique influence la sphère privée et vice versa $»^{13}$.

8 Dans ce jeu de miroirs, la personnalité de l'individu, et donc sa vie quotidienne, devient la somme des actions concrètes pratiquées dans le monde réel et du partage de contenus mis en ligne : la perception du moi dépend de la représentation construite à travers les différentes connexions. Si cela est vrai pour les utilisateurs de Facebook, cela est encore plus vrai pour les affiliés à la mafia. En fait, on oublie souvent qu'entre les organisations criminelles et les médias sociaux, il y a un point de contact : le réseau.

Un réseau matériel et immatériel, qui définit le «positionnement social» (HarreMoghaddam 2003) dans la correspondance entre réel et virtuel: dès lors que l'utilisateur interagit dans la réalité au sein d'une "communauté de pratiques" (Wenger 2006) où les expériences, la culture et la langue sont partagées, il construit un profil numérique qui promeut la pratique mafieuse au plan inter-réel.

10 Comme l'a remarqué Riccardo Scadellari, expert en marketing numérique, "notre identité numérique doit être aussi proche que possible de notre véritable identité. Construire un personal branding ne signifie pas créer un personnage faux et éphémère » ${ }^{14}$. Par conséquent, si l'identité mafieuse réelle prévaut, elle prendra le dessus dans la gestion du personal branding numérique: la crédibilité et l'autorité du profil social découlent de la réputation criminelle de la personne réelle qui conquiert le statut de mafieux inter-réel.

11 Pourtant, à chaque fois que l'actualité a abordé le thème " mafias et Facebook », elle a pris des tons alarmistes en diabolisant l'utilisation du réseau social comme instrument de la «mondialisation mafiogène $»^{15}$. La panique diffusée par les médias révèle la ténacité dans l'imaginaire collectif du "stéréotype progressiste" selon lequel la technologie, produisant des améliorations sociales, culturelles, économiques et civiles, 
avec un parcours linéaire ascendant, marginaliserait les phénomènes de sousdéveloppement comme la mafia, un anachronisme irrationnel incompatible avec l'affirmation d'un contexte dominé par la logique des algorithmes.

12 En fait, comme tout autre utilisateur, les camorristi sont passés par trois phases d'apprentissage méta-technologique, expérimentant à la fois les limites et le potentiel du web participatif. Dans une première période $(2007-2012)^{16}$, ils sont entrés dans le web de manière ludique pour se joindre à la cohorte des gens influençables ${ }^{17}$, mais n'étant pas encore très habiles, ils ont mis en danger la règle d'or de l'omertà. Un certain nombre de fugitifs qui ne connaissaient pas bien la pratique de la géolocalisation ont été arrêtés : la publication de photos personnelles a permis à la police postale de les localiser ${ }^{18}$. Mais une première ébauche d'imaginaire "social mafieux " s'est répandue : dans cette phase, des groupes, des fan pages et des profils fake ont été créés qui, d'une part, rendent hommage aux exploits de grands chefs de clans $\mathrm{du}$ passé et, d'autre part, exaltent la puissance des organisations criminelles du présent $^{19}$.

Cela conduit à une deuxième phase de consolidation (2012-2016), avec la mise en place d'une rhétorique mafieuse spécifique. Les jeunes camorristi apprennent notamment à exploiter le socialcasting, un cadre « où le processus de distribution fait référence à une communauté de personnes qui décident en toute autonomie d'accroître la circulation de contenus grâce aux possibilités de partage offertes par les nouvelles plateformes technologiques $»^{20}$.

L'inter-réalité de la Camorra est maintenant consolidée, avec ses messages textuels et ses fragments audiovisuels explicites ${ }^{21}$, et se manifeste concrètement à travers le courtcircuit réel-virtuel.

Le 16 octobre 2014, Fabio Orefice est blessé lors d'un règlement de comptes entre les clans du quartier Traiano à Naples. Pas du tout intimidé, il défie ses agresseurs et affiche sur Facebook des phrases sans équivoque : « le lion est blessé mais pas mort, je suis déjà debout. Ouvrez bien les yeux ; pour les fermer il suffit d'un rien. Avita muriiii ${ }^{22}$ ». Le message est accompagné de photos montrant les parties du corps où il a été blessé. Il ajoute également des images d'armes et de munitions. Six jours plus tard, deux inconnus, à bord d'une grosse moto, tirent des rafales de kalachnikov sur le portail de son domicile ${ }^{23}$. Autrement dit, le clan rival, follower du profil d'Orefice, réagit réellement à ses menaces virtuelles. Le vécu du camorrista conditionne son identité numérique, qui à son tour est soumise à l'influence de la réalité criminelle. L'interréalité mafieuse conjugue donc le réel et le virtuel, et créé une nouvelle dimension spatiale et temporelle de la criminalité organisée.

16 C'est là que s'ouvre la troisième phase, la phase actuelle, dominée par la «Google generation criminelle ${ }^{24}$, la génération numérique de ceux qui sont nés au milieu des années 1990 et qui savent exploiter le potentiel des réseaux sociaux de manière intuitive et sans effort. Ce sont des jeunes qui vivent dans le liquide amniotique de l'inter-réel, mélangeant les expériences déviantes et les exploits des villains du cinéma, de la télévision ou des jeux vidéo. Ils vivent un processus d'acculturation criminelle méta-technologique basé sur le partage en ligne de façons de parler et de s'habiller, de postures corporelles à tenir, d'armes à utiliser, d'objets cultes à posséder, de phrases à retenir, de photos à partager, de dialogues à transmettre, de clips à regarder. Ce sont les prosommateurs ${ }^{25}$ de l'épique mafieuse, dont la perception de l'expérience est renversée par le va-et-vient continu entre réel et imaginaire. Il a été noté que «les 
jeunes camorristi sont fous de Facebook », qu'ils sont « les parrains de la Camorra 2.0 » et qu'ils utilisent "les réseaux sociaux comme une arme » pour transmettre "des messages mafieux et des déclarations d'intention ${ }^{26}$.

À vrai dire, comme tous ceux de la génération numérique, ils tirent le meilleur parti du potentiel du média, en exploitant des modes d'utilisation inconnus des générations précédentes. Il arrive donc que les mafieux plus âgés demandent aux jeunes recrues de contrôler les mouvements de leurs ennemis en suivant les profils Facebook ou en utilisant la transmission en direct pour communiquer avec le réseau des «amis ${ }^{27}$.

Pour répondre aux questions initiales, il faudra donc entrer dans les méandres de ce monde numérique qui reflète le réel. Nous avons effectué une recherche en activant un faux profil sur Facebook avec une stratégie spécifique de impression management ${ }^{28}$ afin d'interagir avec des protagonistes de la saison dite des stese $e^{29} .80$ profils de jeunes déviants ou borderline âgés de 14 à 24 ans ont été examinés. Le réseau de contacts a été développé à partir de 5 noms parus dans des articles de journaux locaux en 2017 en lien avec des événements criminels d'ordre mafieux ${ }^{30}$. Le réseau relationnel est le premier aspect pertinent: les amis numériques sont choisis sur la base d'une orientation cognitive de nature affective qui influence la composante évaluative. Essentiellement, des liens sont établis entre des personnes réelles qui partagent des émotions et des valeurs dans une communauté fermée de type local (parochial) et parental qui reproduit la dynamique du contexte criminel $^{31}$. La stratégie d'identification est imprégnée de «culture du narcissisme » (Lasch, 1992), exaltée par la pratique du selfie. Ils montrent leurs visages et leurs corps dans des attitudes glamour en utilisant un langage fait de clichés (tirés de films, de fictions, de chansons, de livres, de maximes de personnages célèbres, etc.), copiés sur le web et resémantisés par le couplage d'images et de mots. Songeons par exemple à Francesco, visage d'adolescent, qui se montre sur le seuil de sa maison. Mettant en évidence sa tenue, il écrit : « Tire-moi dessus mais ne te trompe pas car si c'est à moi, je vous ferai mal », ce qui est la version détournée d'un couplet de la chanson Sparami ${ }^{32}$ du rappeur Baby $\mathrm{K}$ : "Tire-moi dessus mais ne te trompe pas / si c'était à moi, je te ferais mal ». Il va de soi que cette phrase, postée sur le profil d'un affilié au clan Fraulella de Ponticelli, a une valeur complètement différente du texte original, qui exprime la rébellion d'une femme contre le machisme. Dans $90 \%$ des cas, ces jeunes hommes portent des vêtements coûteux d'une marque spécifique (Dsquared2) affichée comme élément qualifiant. On pense d'abord qu'ils veulent démontrer leur réussite personnelle (déviante/criminelle) par l'étalage de vêtements exclusifs, en ce sens que ceux qui ne les portent pas n'appartiennent pas à leur monde. Mais là n'est qu'une partie du processus d'identification sociale. Si l'on regarde de plus près, on remarque que dans les informations de profil, à la rubrique "profession", paraissent les mots «travaille chez Dsquared2" ou "manager chez Dsquared2». Qu'est-ce que cela est censé signifier? Vraisemblablement, que le brand commercial symbolise métaphoriquement l'appartenance à un brand social. On se pavane avec un vêtement réservé à une élite pour souligner son appartenance à une organisation sélective, à laquelle seul un petit nombre peut adhérer. Qui plus est, on impose une hiérarchie nette entre ceux qui se contentent de "travailler » et ceux qui ont le rôle de "manager». Les narcotrafiquants mexicains utilisent également des vêtements et certaines marques pour indiquer leur affiliation à un cartel ${ }^{33}$. Sans oublier que le style mafieux a conquis une place de choix sur le marché du luxe ${ }^{34}$. Cependant, en enquêtant sur le présent numérique, l'historien ne peut qu'observer que cette autoreprésentation renvoie à une supériorité morale découlant de l'appartenance à une organisation 
criminelle élitiste. Le camorrista (ou toute personne proche du clan) n'est pas un criminel ordinaire. Au début du XXe siècle déjà, Ferdinando Russo et Ernesto Serao écrivaient: "Les camorristi ont eu, dans le passé, des vêtements et des styles d'habillement particuliers, afin de se reconnaître facilement entre eux $»^{35}$. Il s'agit donc d'une pratique de reconnaissance mutuelle dans la dimension inter-réelle, qui utilise des critères d'identification traditionnels dans un environnement numérique.

Une autre pratique est restée inchangée dans la transition du réel au virtuel: l'affichage de tatouages. Tandis que dans la réalité la peau dessinée n'est visible qu'aux membres de son groupe, sur Facebook elle devient une attestation publique d'identité, un marqueur gravé directement sur la peau. Il en va de même avec les $t$-shirts : les jeunes d'un clan du quartier Sanità de Naples exhibent sur leur poitrine le mot LOVE, où la première lettre est un pistolet, la deuxième une grenade, la troisième un rasoir ouvert, la quatrième une kalachnikov. Aujourd'hui encore, comme par le passé (De Blasio 1897), la pratique consistant à graver les noms d'amis décédés ou des images pieuses est très répandue, mais à cela on ajoute des personnages inattendus comme le Joker ou Benito Mussolini. Dans le premier cas, il s'agit de signaler explicitement la place que l'on occupe dans la société : conscients de jouer un rôle négatif, ces jeunes affiliés se moquent de ceux qui sont de l'autre côté de la barrière ; dans le second cas, il n'y a pas de référence idéologique: le duce incarne la capacité à commander, à maintenir l'ordre par la violence. Dans cette optique, le dictateur est perçu comme un "grand boss » du passé qui a su conquérir le pouvoir en vainquant ses ennemis. En effet, les maximes mussoliniennes que l'on trouve sur la page de Ciro Marfé ${ }^{36}$, que celuici partage sous forme de mèmes ${ }^{37}$, sont dénuées de sens politique mais représentent autant de messages d'avertissement adressés aux clans rivaux. Le 26 août 2015, Marfé publie une photo, avec une phrase superposée : «Si le destin est contre nous, tant pis pour lui »; le 11 avril 2016, il ajoute l'image d'une plaque où est gravé un aphorisme du dictateur: «Nous ne voulons pas la guerre, mais nous ne la craignons pas $»^{38}$. Le 7 février 2016, Walter Mallo, jeune chef émergent du quartier de Miano, lui oppose un mème de Fidel Castro : « ¡ Patria o Muerte, Venceremos! »"

L'affichage de la violence comme métaphore du pouvoir est au centre de la construction de l'imaginaire social de la Camorra. Dans les photos de profils que nous avons examinées on trouve soit des boss ayant existé soit des duplications médiales, mais également des images allégoriques : la lionne, le feu, les armes et des personnages imaginaires. Dans quelques cas (18 profils sur 80 ), les contenus les plus explicites sont partagés avec des profils fake : «Enrico Escobar (El Chapo) »; « El Padron Del Mal (NotRivals)»; «Tony Escobar (Cosa Nostra)»; «Loco Escobar». On aura compris quel impact a pu avoir la série télévisée à succès Narcos et combien le mythe de Tony Montana joué par Al Pacino dans le film Scarface (1983) est encore important, après plus de trente ans.

21 Les utilisateurs déviants composent un imaginaire fragmenté mais organique : dans le web, ils puisent des images de nature différente (cinéma, télévision, blogs, sites, réseaux sociaux) et les rassemblent en les adaptant à leur propre personal branding, avec un impression management à caractère criminel. Il peut donc arriver qu'avec Totò Riina, Raffaele Cutolo, Michele Zagaria et Pablo Escobar, on trouve Tony Montana, Jenni Savastano, 'O Trac (un des personnages de la série Gomorra), Michael Corleone, Ben Laden et Abu Bakr al-Baghdadi. 
Un scénario globalisé dans lequel la réalité et l'imagination, la Camorra et le terrorisme se confondent pour produire un message violent original qui peut être partagé sur le net. La référence au terrorisme n'est pas non plus nouvelle. Dans un jugement du Tribunal de Naples, au début des années 1980, on peut lire: la Nouvelle Camorra Organisée (c'est-à-dire les clans dirigés par Raffaele Cutolo) est le « terrorisme de notre sous-prolétariat qui, se livrant à un qualunquismo politique absolu, retrouve son identité en tant que masse $»^{40}$. Sans oublier que déjà après l'Unification, la Camorra a été identifiée comme une "secte" issue des "classes dangereuses", "criminelle et subversive $»^{41}$. La référence actuelle à Daech signale l'assimilation de l'imaginaire globalisé et contribue, avec l'utilisation sans pudeur et sans limites d'images terrifiantes, au renforcement numérique du storytelling camorriste. Voici un exemple : le 17 octobre 2016, Diego Otavimenna est arrêté par les carabiniers, dans un village de l'arrière-pays napolitain, pour incitation au crime, avec la circonstance aggravante d'apologie du terrorisme. Responsable d'une quincaillerie, trentenaire, Otavimenna a affiché sur son profil Facebook une vidéo montrant la décapitation d'un prisonnier de Daech, accompagnée de quelques phrases de soutien à la Camorra ${ }^{42}$.

23 L'exemple le plus approprié, en ce sens, concerne le clan des Barbudos du quartier Sanità. Ce nom semble inspiré des trafiquants de drogue sud-américains, mais dans leurs attitudes et leurs annonces, les affiliés font référence aux djihadistes. Sur leurs profils Facebook, ils montrent des tatouages en arabe, se présentent avec des noms de bataille qui rappellent ceux des miliciens et postent des citations du genre « Je suis le dernier élu », ou bien ils écrivent : « Je suis comme Daech, eux, ils avancent en tenant la kalachnikov, moi, un simple couteau, eux, ils tirent dans la tête, moi, je ne donne qu'un coup de couteau dans la gorge $»^{43}$.

24 Est-il possible que les jeunes de la Camorra, en surfant sur le web, se soient identifiés aux extrémistes islamiques, transformant la marginalisation sociale en une sorte de fondamentalisme criminel? Songeons par exemple à Emanuele Sibillo, chef de la paranza des enfants ${ }^{44}$, assassiné le 2 juillet 2015 dans une embuscade, à l'âge de 19 ans. Entre 2014 et 2015, il a dirigé la faction qui s'opposait aux anciens clans du centre historique de Naples, à partir du quartier Forcella. Emanuele utilise Facebook pour diffuser sa propagande armée : le réseau de ses contacts reproduit le réseau criminel. Sur son profil, il affiche des pensées et des contenus multimédia sans crainte d'être géolocalisé. Tous les affiliés de la paranza ont tatoué sur leur bras gauche le chiffre 17 entouré de flammes, $\mathrm{S}$ étant la dix-septième lettre de l'alphabet italien: S comme Sibillo. Sur les murs du quartier apparaissent les initiales FS (Famille Sibillo) pour marquer le territoire. Il répète souvent à sa mère qu'il n'atteindra pas l'âge de 20 ans, mais qu'il n'a pas peur de la mort ${ }^{45}$. Il est le leader d'une "guerre sainte» dont l'objectif est moral, dans une logique de profit : « récupérer » le quartier pour contrôler le marché. On se bat pour conquérir une allée, un immeuble, une place. Le conflit exige que l'on ait une identité particulière afin de radicaliser la diversité de l'adversaire. Il faut écraser, soumettre et humilier l'ennemi pour affirmer sa supériorité. S'enclenche ainsi un mécanisme d'action et de réaction, donnant lieu à une chaîne infinie de meurtres où, chacun à son tour, on prend le dessus. La lutte se transforme en guérilla urbaine permanente, un conflit sans règles qui bouleverse la ville et fait nombre de victimes innocentes. Entre vie réelle et vie virtuelle, Emanuele construit le mythe du boss inter-réel et fait son entrée dans la culture de masse, par le biais de la presse bien sûr, mais aussi grâce à la littérature et au cinéma : l'histoire de la paranza des enfants a 
inspiré jusqu'ici trois romans, un documentaire et un film ${ }^{46}$. Mais ce n'est pas tout. Sibillo devient aussi un personnage de l'imaginaire populaire. Dans une photo prise lors du Carnaval de 2016, un enfant âgé d'une dizaine d'années porte un costume croisé rayé et une fausse barbe aussi longue que celle d'Emanuele. Il pose à côté de l'image de Saint-Janvier, devant laquelle on peut remarquer deux compositions florales en forme de 17 et de FS. Or, associer Saint-Janvier, l'enfant gangster barbu et les codes chiffrés de la paranza revient à superposer le saint patron de la ville et Emanuele Sibillo. Le petit boss, du haut des cieux, protège le quartier de Forcella. La photo en question a été postée sur un faux profil au nom de Fortunato Di Venanzio, âgé de quinze ans, frère d'un mafieux en cavale, Rodolfo, personnage de la série télévisée L'onore e il rispetto. En parcourant l'album photo du profil, on voit se suivre les images d'Emanuele « élevé au rang d'ange gardien vers qui on peut se tourner en utilisant le réseau social $\aleph^{47}$. L'exemple de Sibillo démontre d'une part que la violence crée le lien entre le réel et l'imaginaire, et d'autre part que le sentiment de fraternité est utilisé pour défendre la communauté familiale-criminelle :

Quand on est frère de sang, impossible de revenir en arrière. Les destins s'unissent aux règles. On vit et on meurt suivant sa capacité à rester dans le cadre de ces règles. La 'Ndrangheta a toujours opposé le frère de sang au frère de péché, c'est-àdire le frère que la mère vous donne en péchant avec votre père. Le frère qu'on se choisit, qui n'a rien à voir avec la biologie, qui ne naît pas d'un utérus, ne vient pas d'un spermatozoïde. Celui qui naît du sang ${ }^{48}$.

Ce lien est reproduit sur Facebook grâce à la publication de photos qui correspondent au pacte : un baiser sur la bouche associé à l'emoji ${ }^{49}$ représentant une seringue avec une goutte de sang. C'est la métaphore de la transfusion qui génère un partage des affects et des valeurs extra-familiales au sein de la communauté restreinte à laquelle on appartient. De l'image unie au texte se dégage une intonation typique de l'oralité, une qualification conceptuelle qui renforce l'empathie collective au sein du réseau relationnel. En plus de la seringue qui saigne, les symboles les plus couramment utilisés sont : la bombe qui explose, le pistolet, le couteau, le poing (ou le poing américain), le missile, l'ange, la croix, la tête de mort, le fantôme, la feuille de marijuana, les trois singes, les icônes TOP 100 et plusieurs autres souvent associés à l'image du cœur. Chaque image a une signification différente selon le destinataire du post. On peut utiliser les mêmes symboles mais la signification du message change en fonction de l'interlocuteur: l'ami, l'ennemi, le traître, le sournois, le lâche, le gentil, le garçon comme il faut, le gang, la fiancée, la maîtresse, etc. De plus, la fréquence de certains symboles entrâne une re-sémantisation numérique du jargon mafieux: les émoji représentant des armes (bombes, pistolets, couteaux, missiles, etc.) appartiennent à l'iconographie des jeux vidéo; les références à la fraternité (poing, sang, feuille de marijuana, etc.) proviennent de la sphère du hip hop ; d'autres viennent de la tradition populaire (tête de mort, fantôme, trois singes) ou du symbolisme catholique (ange, croix). L'ensemble des images, avec l'ajout du texte, modifie les intentions du message qui, la plupart du temps, est banal, stéréotypé et copié d'autres profils. Un amas d'objets et de concepts qui confère à la séquence symbolique le caractère de communication de type criminel. Les symboles du clavier acquièrent ainsi une fonction polysémique qui renvoie tant à la mentalité individuelle qu'à celle de la communauté de référence: l'hybridation des mots et des signes est une pratique innovante par rapport à l'ancienne pratique criminelle du jargon ; une innovation propre à la Google generation, qui utilise l'emoji pour tracer les frontières virtuelles de l'éthique mafieuse. Les symboles sont des outils de contextualisation à forte résonance empathique : ils 
soulignent les émotions de l'écrivant en simulant un geste, une expression faciale, une inflexion de la voix ou une posture du corps qui ne peuvent être ignorés. Par conséquent, le langage non verbal est essentiel dans le symbolisme de la mafia numérique. On peut aller jusqu'à affirmer qu'elle se renouvelle grâce à la simultanéité, l'efficacité et l'exemplarité de symboles qui transmettent des sensations difficiles à exprimer avec des mots. Les emojis sont au centre d'un système de communication bidirectionnel : ils "parlent» au groupe des jeunes affiliés ou borderline (niveau intracommunicatif) et envoient des messages de menace au monde extérieur (niveau extracommunicatif). En ce sens, ils font fonction de médiateurs entre la mentalité de la Camorra et la culture locale et nationale.

La fraternité est donc au cœur de la rhétorique symbolique et textuelle des camorristi numériques. En publiant le baiser, ils relient le réel au virtuel en dessinant l'image d'un groupe criminel inter-réel que chacun doit connaître. Celui avec qui on partage la dangerosité du « métier », est un compagnon dans les deux mondes : on l'appelle bro', à savoir brother, qui a une signification plus forte que le traditionnel compare [partenaire]. La Google generation de la Camorra ressent l'urgence de montrer au public connecté à quoi ressemble la vie damnée des niggers italiens, ou plutôt des jeunes violents prêts à partager, au même titre que les "frères américains", la violence de rue, le contrôle du territoire, le trafic de drogue, la fierté du clan, la mort précoce et même la passion du hip hop. Ce n'est pas un hasard si, sur leurs profils, le partage de chansons napolitaines « mélodiques » est en baisse tandis que la diffusion de morceaux de rap ou de trap ${ }^{50}$, accompagnés de photos de Tupac Skahur ou de gangs urbains, est en hausse.

Sur l'un des profils fake consacrés à Michele Zagaria, chef du clan des casalesi, par exemple, on a publié la photo d'un jeune noir, un pistolet à la main droite, tenant un Évangile de la main gauche. Cela signifie que la « main du travail », la droite, peut tirer, tandis que la "main du cœur", la gauche, est consacrée à la prière. La version originale $^{51}$ de cette photo fait partie du répertoire du site www.artcoup.com, qui contient, parmi tant de scènes de la vie réelle, celles où paraissent les gangs jamaïcains de Kingston ${ }^{52}$. Cette synthèse iconographique, re-postée sur ce profil fake, change complètement de sens : d'une part, elle force à associer mentalement la traditionnelle compatibilité entre Camorra, religion et violence; d'autre part, elle montre une réelle volonté d'auto-représentation de son identité personnelle dans le cadre d'un récit social où l'image du jeune camorrista est assimilée à celle du criminel ethniquement typé. D'ailleurs, tout comme les noirs des ghettos, ces adolescents transforment la langue en slang, à partir du clavier Qwerty, en faisant fusionner l'identité criminelle avec l'identité territoriale. Leur langue n'est pas le napolitain de Salvatore Di Giacomo ou d'Eduardo De Filippo, ni la langue vernaculaire de l'ancienne capitale du Royaume des Deux-Siciles, ni le dialecte créolisé de Raffaele La Capria ${ }^{53}$. Il s'agit plutôt d'un idiome glocal qui unit le territoire urbain, la communauté locale et la mentalité criminelle à la sphère des new media (tout en intégrant l'imaginaire des old media). Si on voulait mieux définir cette langue de la Google generation, on pourrait dire qu'elle relève du napolitanisme plus que d'un parler authentiquement napolitain. L'imaginaire et le jargon de ces jeunes violents est la manifestation plastique d'un fondamentalisme culturel fermé à la confrontation entre identité locale et dimension nationale, mais en même temps ouvert aux influences de la mondialisation numérique. Ils sont enfermés dans une ville de Naples étriquée, écrasée entre les ruelles de Forcella et les forts de 
Ponticelli et San Giovanni. L'Italie, quant à elle, est une terre étrangère, haïe, qui emprisonne et dénature les « frères » les plus faibles, incapables de se frayer un chemin dans la métropole tentaculaire.

Qu'est-ce le napolitanisme? C'est un fondamentalisme qui trace une frontière nette entre le «dedans » et le "dehors », qui lie l'identité territoriale à l'identité marginale ou déviante, transformant le complexe victimaire du Sud de l'Italie si mal aimé après l'Unification en fierté criminelle inscrite à plein titre dans la mondialisation. Dans la dimension inter-réelle où évoluent les jeunes camorristi, la modernité de la mondialisation coexiste avec la structure anthropologique et psychologique classique de la mafia : les membres de l'organisation restent fidèles, malgré tout, à leur identité sociale qui justifie la coercition et l'usage brutal de la violence comme instrument de pouvoir ${ }^{54}$. Cette obsession confirme l'existence d'une identité qui se forme dans une "pensée déjà pensée ", d'une identité structurée par la longue sédimentation du contexte camorriste.

Sur Facebook se dessine la dichotomie entre une perception " primitive » qui sépare le « nous social» (l'ennemi) du «nous familial » (l'ami). La Google generation reproduit le modèle de l'obéissance a priori et diffuse des pratiques de soumission psychique qui n'admettent aucune pensée divergente, ambivalente, critique ou réfléchie. Ce faisant, elle réaffirme les dynamiques inclusives et absolutistes du militantisme mafieux - un militantisme similaire, de par ses caractéristiques psycho-sociales, aux fondamentalismes religieux ou politiques ${ }^{55}$. Au niveau de l'imaginaire collectif, on brandit ainsi l'idée d'un terrorisme criminel, qui trouve sa justification dans l'idée stéréotypée d'une rédemption sociale violente s'efforçant d'unir la grandeur de Naples (meurtrie par la perte de son statut prestigieux de ville capitale) au pouvoir de la Camorra.

L'analyse des profils Facebook permet de constater que le réseau social, en tant que média, préserve, réplique et communique, tel un amplificateur transgénérationnel, la mémoire culturelle de la Camorra. En l'espèce, on peut parvenir au même constat proposé par Marco Santoro à propos de Cosa Nostra :

Il n'y a aucune raison de croire que les mécanismes psychosociaux, cognitifs et institutionnels qui régissent la formation et la transmission de la "mémoire culturelle » ne s'appliquent pas à la mafia. [...] Mais surtout, en tant que culture statutaire, la mafia est constituée par un système de signes rituels et de structures symboliques qui la représentent comme une entité distincte et identifiable [...] Comme tous les êtres humains, les mafieux ne sont pas de simples créatures matérielles mais des producteurs, des utilisateurs et, naturellement, des manipulateurs de symboles. Et cette production symbolique n'est pas nécessairement une création individuelle ou intentionnelle ; au contraire, elle est le plus souvent collective et non réfléchie. [...] Les termes d'honneur, de loyauté, de trahison, d'amitié, de même que la violence dans ses différents modes d'expression - qui sont essentiels au lexique et à l'univers culturel mafieux - font référence à cette structure symbolique qui organise le monde et à la perception qu'en a le mafieux : une structure symbolique qui accorde une place à la créativité sociale ainsi qu'aux idiosyncrasies individuelles ${ }^{56}$.

31 L'aspect glamour de la Camorra et la répétition de certaines icônes restituent une image de l'organisation criminelle en tant que communauté esthétique et affective : le partage de vêtements, d'accessoires, d'objets, rituels et symboles rappelle l'adhésion aux valeurs de la communauté qui caractérise son identité de façon esthétique. En d'autres termes, on établit une correspondance symbolique entre des biens de nature 
économique ou commerciale et des liens de nature morale et esthétique. Une correspondance qui, au sein de la communauté déviante elle-même, génère « une série de significations sociales et personnelles qui sont directement liées aux domaines du sentiment, de la représentation de soi et de l'image morale, de sorte que la distinction entre un bon produit et un mauvais produit n'est pas strictement régie par des valeurs monétaires ni même par la concurrence, mais par d'autres éléments fortement conditionnés par une identité culturelle commune $\aleph^{57}$. Cette identité est le pivot autour duquel tourne la rébellion esthétique qui transforme la honte de la stigmatisation sociale en fierté criminelle :

Comme les ghettos ethniques et raciaux, ces communautés représentent un paradis d'autodéfense et le lieu où l'individu déviant peut prétendre ouvertement être au bas de l'échelle comme tout le monde. En outre, les déviants sociaux ont souvent la certitude d'être non seulement comme les gens normaux, mais même meilleurs, et que la vie qu'ils mènent est bien meilleure que la vie à laquelle ils étaient prédestinés. De plus, les déviants sociaux offrent des modèles de vie aux normaux insatisfaits, parvenant ainsi à attirer leur sympathie, mais aussi à faire les prosélytes $^{58}$.

Cette attitude est manifeste dans l'agressivité du profil de Lello, rejeton d'une importante famille de la Camorra ${ }^{59}$. Bien qu'assigné à résidence, il reste en contact avec son réseau et écrit : «La défense doit toujours être légitime : voilà notre CODE !». Il ajoute la photo d'un collier avec un pendentif en forme de pistolet ${ }^{60}$. Après avoir annoncé la fin de l'assignation à résidence - « Bonjour tout le monde, libre à nouveau, n'en déplaise à toutes les putes et à tous les cocus qui me voulaient du mal, à moi et aux miens $»^{61}$ - il publie une photo de ses derniers achats : des chaussures, des pantalons et des $t$-shirts de la célèbre marque, en quantité disproportionnée ${ }^{62}$. Deux jours plus tard, revenu à la routine, il partage la phrase suivante : «Comme c'est agréable de se sentir maitre de soi, aimé par tant d'amis et respecté avec respect mutuel par ceux qui méritent que je les salue, mais le mieux, c'est de voir tant de chiens de berger baisser les yeux en croisant mes yeux $»^{63}$. Un message sans équivoque pour réaffirmer son rôle de leader, souligné par l'ajout de deux emoji en forme de pistolet et de cinq bras en tension musculaire. Les mots et les images forment un récit dans lequel ses semblables peuvent se reconnaître, en parcourant un chemin symbolique préétabli : la Camorra impose les stigmates du pouvoir, mais le commandement est aussi une question de style.

En guise de conclusion, on peut observer que le "regard historique " porté sur le présent numérique a permis de repérer une identité collective non éphémère, établie grâce à l'activation d'une mémoire culturelle qui réagit à la stigmatisation sociale avec la fierté de la diversité. En même temps, ce regard a permis de constater que l'activisme social implique une conversion au conformisme de masse: ces jeunes, comme tout autre internaute, sont attirés par la logique des réseaux sociaux qui a transformé les utilisateurs en biens à vendre sur le marché :

Ils sont à la fois les marchands et les marchandises dont ils font la promotion. Ils sont la camelote et le camelot [...]. Tous, quelle que soit la catégorie dans laquelle les compilateurs de statistiques les placent, vivent dans le même espace social qu'on appelle le marché [...] l'activité à laquelle ils se consacrent est le marketing. Le test qu'ils doivent passer pour aspirer à la reconnaissance sociale qu'ils désirent les force à se transformer en marchandises, en produits d'appel susceptibles de générer une demande et d'attirer les clients ${ }^{64}$. 
Suivant les traces de l'imaginaire numérique, on peut en somme affirmer que la Google generation criminelle utilise Facebook sans renier son passé. Bien au contraire, elle le réaffirme en construisant une mémoire culturelle inter-réelle mondialisée, qui suit la voie du marketing des médias sociaux.

\section{BIBLIOGRAPHY}

Bauman, Zygmunt et Lyon, David (2013), Sesto potere. La sorveglianza nella modernità liquida, RomaBari, Laterza.

Benigno, Francesco (2015), La mala setta. Alle origini di mafia e camorra 1859-1878, Torino, Einaudi. Bennato, Davide (2011), Sociologia dei media digitali, Roma-Bari, Laterza.

Ceccarini, Luigi (2015), La cittadinanza online, Bologna, Il Mulino.

De Blasio, Abele (1897), Usi e costumi dei camorristi. Storia di ieri e di oggi, Napoli, Edizioni Pierro.

Goffman, Erving (2010), Stigma. L'identità negata, Verona, ombre corte.

Fiore, Innocenzo (1997), Le radici inconsce dello psichsimo mafioso, Milano, Franco Angeli.

Gruzinski, Serge (2015), L'histoire pour quoi faire ?, Paris, Fayard.

Harre, Rom - Moghaddam, Fathali M, éd. (2003), The Self and Others : Positioning Individuals and Groups in Personal, Political and Cultural Contexts, Westport, Praeger.

Lasch, Christopher (1992), La cultura del narcisismo. L'individuo in fuga dal sociale in un'età di disillusioni collettive, Milano, Bompiani.

Lo Verso, Girolamo, éd. (1998), La mafia dentro. Psicologia e psicopatologia di un fondamentalismo, Milano, Franco Angeli.

Lo Verso, Girolamo (2013), La mafia in psicoterapia, Milano, Franco Angeli.

Mininni, Giuseppe, éd. (2002), Virtuale.com. La parola spezzata, Napoli, Idelson-Gnocchi.

Pesce, Anita \& Stazio, Marialuisa (2013), La canzone napoletana. Tra memoria e innovazione, Napoli, Cnr-Issm.

Riva, Giuseppe (2014), Nativi digitali. Crescere e apprendere nel mondo dei nuovi media, Bologna, Il Mulino.

Riva, Giuseppe (2012), Psicologia dei nuovi media. Azione, presenza, identità e relazioni nei media digitali e nei social media, Bologna, Il Mulino.

Russo, Ferdinando et Serao, Ernesto (1907), La camorra. Origini, usi, costumi e riti dell'« annorata società », Napoli, Bideri.

Santoro, Marco (2007), La voce del padrino. Mafia, cultura, politica, Verona, ombre corte.

Saviano, Roberto (2016), La paranza dei bambini, Milano, Feltrinelli (Pirhanas, traduit de l'italien, par Vincent Raynaud, Paris, Gallimard, 2018).

Cahiers de Narratologie, 36 | 2019 
Scandellari, Riccardo (2014), Fai di te stesso un brand. Personal branding e reputazione online, Palermo, Flaccovio.

Van Kokswijk, Jacob (2003), Hum@n, Telecoms, \& Internet as Interface to Interreality, Hoogwoud, Bergboek.

Wenger, Etienne (2006), Comunità di pratica. Apprendimento, significato e identità, Milano, Raffaello Cortina Editore.

Wright, Robert (2000), Non-Zero: The Logic of Human Destiny, New York, Pantheon.

\section{NOTES}

1. Gruzinski 2015, p. 18.

2. Ivi, p. 20.

3. Idem.

4. Ivi, pp. 23-24.

5. Ivi, p. 24.

6. Idem.

7. Cf. https://bit.ly/2siZC7m, consulté le 30 novembre 2018. Sauf précision contraire, toutes les citations en langue étrangère sont livrées en version française par la traductrice de ce texte.

8. Cf. https://bit.ly/2vxwpXI, consulté le 30 novembre 2018.

9. T. Cantelmi, « Educare nell'era digitale e tecnoliquida », https://bit.ly/2F3ppWD, p.1, consulté le 30 novembre 2018.

10. Riva 2012, pp. 22-23.

11. M. Prensky, « Digital Natives, Digital Immigrants », https://bit.ly/2BR4LEU, consulté le 30 novembre 2018

12. Riva 2014, p. 62.

13. Id., p. 60.

14. Scandellari 2014, p. 32.

15. U. Santino, Modello mafioso e globalizzazione, https://bit.ly/2TpKo9e, consulté le 30 novembre 2018.

16. C'est la période du développement de Facebook en Italie : on passe de 200.000 à plus de 20 millions d'utilisateurs.

17. Cf. "La camorra di Pomigliano cerca adepti : Gruppo su Facebook fondato da giovani », Corriere del Mezzogiorno, 28 septembre 2009.

18. Ravveduto 2015, p. 195.

19. A. Meccia, «Ipse dixit. L'abbecedario di Totò Riina ", 9 septembre 2010, https://bit.ly/ 2F3jSPW, consulté le 30 novembre 2018.

20. Bennato 2011, p. 6 .

21. E. Ciaccio, «Camorra, il boss grida vendetta su Facebook», 17 août 2012, https://bit.ly/ 2As15JY, consulté le 30 novembre 2018; S. Di Meo, «Baby-camorrista, sparò a una volante della polizia. In posa su Facebook con fucile e coltello», 13 janvier 2014, https://bit.ly/2R3SG9S, consulté le 30 novembre 2018; A. Balestra, «Camorra. Il boss torna libero in permesso premio, sfida tutti su Facebook », 8 mars 2015, https://bit.ly/2QitXti, consulté le 30 novembre 2018.

22. Dialecte napolitain : « Vous devez crever » $(N d T)$.

23. "Camorra, lo feriscono: lui li minaccia e li sfida su Fb. Loro tornano a sparargli », La Repubblica, 23 octobre 2014, https://bit.ly/2RnlGZR, consulté le 30 novembre 2018; F. Feo, «Camorra, la "posta" dei clan», 19 novembre 2017, https://bit.ly/2s9eT7S, consulté le 30 novembre 2018. 
24. M. Ravveduto, «La paranza dei bambini. La Google Generation di Gomorra », Questione Giustizia, 14 gennaio 2017, https://bit.ly/2shofyt, consulté le 30 novembre 2018.

25. Prosommateur est un néologisme issu de la contraction de producteur/professionnel et consommateur (de l'anglais prosumer : producer/professional + consumer).

26. C. Caprio, "Perché i giovani camorristi vanno pazzi per Facebook», Vice, 10 août 2016, https://bit.ly/2RuDNwV, consulté le 2 décembre 2018; C. Zagaria, «I giovani padrini della camorra 2.0: social usati come un'arma » La Repubblica, 6 mai 2016, https://bit.ly/2VuJZnR, consulté le 2 décembre 2018 ; G. Longo, « Camorristi di quarta generazione : kalashnikov, tatuaggi e Facebook », La Stampa, 7 septembre 2015, https://bit.ly/2BZvHT9, consulté le 2 décembre 2018. 27. D. Del Porto, «I boss : "Cerca i nomi su Facebook". Caccia ai nemici da colpire sui profili Social ", La Repubblica, 28 juin 2016, https://bit.ly/2BXVvPv, consulté le 2 décembre 2018; "Camorra, l'ex boss pentito in diretta Facebook: "Chi è contro di noi deve schiattare" ", Corriere del Mezzogiorno, 10 octobre 2018, https://bit.ly/2s6V3tK, consulté le 2 décembre 2018.

28. J. Rosenberg - N. Egbert, « Online Impression Management: Personality Traits and Concerns for Secondary Goals as Predictors of Self-Presentation Tactics on Facebook », Journal of ComputerMediated Communication, 17, 2011, https://bit.ly/2sic3gH, consulté le 2 décembre 2018. La stratégie d'impression management d'un profil fake s'organise autour du partage de photos, de vidéos, de post de film et de fictions sur les mafias ou sur la vie de chefs mafieux. Le partage comprend aussi des chansons napolitaines melodiche, des images pieuses, des photos : de l'équipe de football de Naples, d'armes, de vêtements à la mode, de voitures et de moto de sport, d'objets de luxe.

29. La stesa est une pratique utilisée par les jeunes de la Camorra pour semer la terreur dans les quartiers de Naples. Lancés à toute vitesse sur des scooters, ils tirent des balles en l'air ou contre des sièges d'entreprises pour répandre la terreur et défier les clans rivaux sur leur territoire. La réaction qui suit ce genre de raids est donc la stesa, une sorte de pavage humain: les passants sont obligés de s'allonger au sol pour éviter de se retrouver dans la ligne de mire des criminels ou d'être touchés par des balles perdues. Cf. notamment R. Saviano, «La paranza dei bambini nella guerra di Napoli », La Repubblica, 8 septembre 2015, https://bit.ly/2H56T1Z, consulté le 2 décembre 2018.

30. Notre recherche a débuté en septembre 2017: s'agissant d'une cover research, nous ne pouvons fournir ni les noms ni les URL rattachés aux profils Facebook cités.

31. Ceccarini 2015, pp. 66-68.

32. "Tire-moi dessus ", NdT.

33. Cf. https://bit.ly/2skuIZd, consulté le 2 décembre 2018.

34. Cf. https://labellamafiaclothing.com/. Il s'agit d'un site de e-commerce brésilien dont le siège est à Miami. Spécialisé dans la vente de vêtements de sport, d'accessoires et de cosmétiques, il utilise les slogans suivants : «Laissez le luxe venir à vous"; "LabellaMafia est plus qu'une marque, c'est un style de vie ", consulté le 2 décembre 2018. Voir aussi l'entreprise Barabas de Los Angeles, qui présente ses chemises portées par El Chapo Guzman, https://bit.ly/2RATUZV, consulté le 2 décembre 2018.

35. Russo - Serao 1907, p. 21.

36. L'identité Facebook de Ciro Marfé peut être dévoilée car il a été assassiné en août 2016, dans une embuscade. La presse a utilisé les posts de son profil pour décrire son activité criminelle. $\mathrm{Cf}$. M. Chiapparino, « Camorra, duplice omicidio a Napoli: "Dopo il raid fuochi d'artificio" », Il Mattino, 4 août 2016.

37. https://bit.ly/2VGZRDS, consulté le 2 décembre 2018.

38. https://bit.ly/2BqsX1D, consulté le 2 décembre 2018.

39. Nous pouvons nommer Walter Mallo car il a été arrêté en mai 2016. Cf. L. Del Gaudio, « Napoli. Preso il boss di Facebook Mallo. Lo sfogo delle mamme al telefono: "Stanno facendo tarantelle grosse..." ", Il Mattino, 6 mai 2016. 
40. Ordonnance-Arrêt Abbagnale Agostino+711, Tribunal de Naples, 1983, p. 87. Le terme qualunquismo (au départ associé à un mouvement politique prônant l'anti-politique, né après la guerre, dans les années 1940) désigne désormais la méfiance et la prise de distance méprisante vis-à-vis des institutions démocratiques, de l'État, des partis traditionnels.

41. Benigno 2015, pp. 33-128.

42. Cf. https://bit.ly/2HVqamQ, consulté le 5 décembre 2018 et https://bit.ly/2UEWN9G, consulté le 5 décembre 2018.

43. Cf. R. Russo, "Il rampollo del boss di camorra e i suoi 5 mila amici su Facebook », Corriere del Mezzogiorno, 20 avril 2016, et M. Ravveduto, «La mafia è una jihad o la jihad è una mafia?", Fanpage, 28 juillet 2016.

44. La paranza dei bambini, littéralement "le clan des enfants", est le titre d'un ouvrage de Roberto Saviano (2016) consacré aux bandes formées de gamins (trad. fr. Piranhas, Paris, Gallimard, 2016).

45. https://bit.ly/2WiQKsz, consulté le 20 novembre 2016.

46. Du roman La paranza dei bambini (cf. note 44), Claudio Giovannesi a tiré le film homonyme, sorti en salle en 2019. Cf. aussi R. Saviano, Bacio feroce, Milan, Feltrinelli, 2017 et S. Di Meo, Gotham city. Viaggio segreto nella camorra dei bambini, Piemme, Milano, 2017, ainsi que le film documentaire de 2018 ES17, en cinq épisodes, écrit par D. Ligorio et C. Sannino, basé sur des textes de R. Saviano.

47. N. Falco, « Camorra, il ragazzino che imita sul web il babyboss ", Il Mattino, 3 avril 2016.

48. Saviano 2016, trad. française 2018, p. 188.

49. Cfr. https://bit.ly/2RDdoP8, consulté le 5 décembre 2018.

50. M. Ravveduto, «Spaccio, omicidi, violenza: alla scoperta della musica "Trap" in terra di

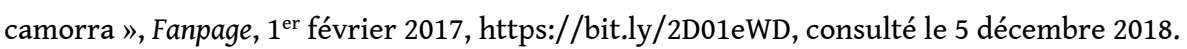

51. Cfr. https://bit.ly/2MRqZvD, consulté le 5 décembre 2018.

52. Cfr. https://bit.ly/2GqcP3E, consulté le 5 décembre 2018.

53. Ravveduto 2007, pp. 30-35.

54. Cf. Lo Verso 2013.

55. Cf. Fiore 1997; G. Lo Verso 1998 ; Lo Verso 2013.

56. Santoro 2007, pp. 44-45, traduit par nous.

57. M. Caiafa, «"E si nun canto moro". L'industria della musica neomelodica a Napoli », in Pesce \& Stazio 2013, p. 459.

58. Goffman 2010, p. 178.

59. A l'heure actuelle, le profil de Lello n'est plus visible pour des raisons de confidentialité.

60. Post publié le 25 juillet 2017 à $2 \mathrm{~h} 24$, sans géolocalisation.

61. Post publié le 4 août à $11 \mathrm{~h} 30$, sans géolocalisation.

62. Post publié le 6 août à $22 \mathrm{~h} 24$, géo-localisé à Naples.

63. Post publié le 8 août 2017 à 06h00, géo-localisé à Naples.

64. Bauman et Lyon 2013, pp. 17-18.

\section{ABSTRACTS}

Cette contribution présente une recherche consacrée à l'utilisation des réseaux sociaux par de jeunes affiliés aux clans de la Camorra ou plus généralement par des jeunes borderline influencés 
par l'imaginaire mafieux. A travers l'analyse qualitative de messages, d'images et du partage de contenus numériques, il retrace l'activisme social de la première Google generation criminelle. Entre vêtements et tatouages, selfies et mèmes, usage argotique de l'emoji ou du hashtag et du live streaming, d'images religieuses et de musique underground (neomelodica-rap-trap), se construit un mush up sous-culturel dans lequel tant les objets que les concepts donnent lieu à une séquence symbolique originale, qui raconte la fierté de la diversité criminelle. Les mots et les symboles acquièrent une fonction polysémantique et parlent autant de la mentalité des individus que de celle de la communauté à laquelle ils appartiennent. L'analyse des profils Facebook montre que la sémantique de la violence reste au centre du code symbolique de la mafia. Ou plutôt, le code est renouvelé grâce à la simultanéité, à l'efficacité et à l'exemplarité de l'information, qui transmet un spectre émotionnel complexe au lieu de concepts empathiques trop difficiles à exprimer avec des mots (surtout pour ceux qui ont un faible niveau d'éducation mais une bonne prédisposition aux «métiers » du numérique). La pratique du sharing est la plaque tournante d'un système de communication bidirectionnel : elle "parle» au groupe des jeunes affiliés ou borderline (intracommunicative) et envoie des messages de menace au monde extérieur (extra-communicative). En ce sens, elle fait fonction de médiateur entre l'imaginaire criminel mondialisé et la mentalité mafieuse locale et nationale, établissant un lien entre deux contextes apparemment distants.

Il saggio presenta una ricerca sull'uso dei social network da parte dei giovani affiliati ai clan di camorra o più generalmente di ragazzi borderline suggestionati dall'immaginario mafioso. Attraverso l'analisi qualitativa dei post, delle fotografie e dello sharing di contenuti digitali si ricostruisce l'attivismo social della prima Google generation criminale. Dall'abbigliamento ai tatuaggi, dai selfie ai meme, dall'uso gergale di emoji e hashtag alle dirette streaming, dalle immagini religiose alla musica underground (neomelodica-rap-trap) emerge un mush up subculturale in cui oggetti e concetti danno luogo a una sequenza simbolica originale che racconta l'orgoglio della diversità criminale. Parole e icone acquistano una funzione polisemantica che racconta sia la mentalità individuale, sia quella della comunità di appartenenza. Dall'analisi dei profili Facebook si può notare come la semantica della violenza rimanga centrale nel codice simbolico mafioso. Anzi, il codice si rinnova grazie alla simultaneità, all'efficacia e all'esemplificazione di informazioni che trasmettono un complesso spettro emozionale in luogo di concetti empatici troppo difficili da esprimere con le sole parole (soprattutto per chi ha un basso grado di scolarizzazione ma una buona predisposizione al "mestiere" digitale). La pratica dello sharing è lo snodo di un sistema di comunicazione bidirezionale: "parla » al gruppo di giovani affiliati e ragazzi borderline (intra-comunicativo) e invia messaggi minacciosi al mondo esterno (extra-comunicativo). In tal senso svolge una funzione di mediazione tra l'immaginario criminale globalizzato e la mentalità mafiosa locale e nazionale, gettando un ponte tra due contesti solo apparentemente distanti.

\section{INDEX}

Keywords: Camorra, Facebook, young criminals, borderline youth, criminal imagination, digital imagination

Mots-clés: Camorra, Facebook, jeunes criminels, jeunes borderline, imaginaire criminel, imaginaire numérique 


\section{AUTHORS}

\section{MARCELLO RAVVEDUTO}

Marcello Ravveduto est enseignant-chercheur en Digital Public History à l'Université de Salerne et à l'Université de Modena et Reggio Emilia. Il est membre du bureau de l'Association italienne de Public History ainsi que du comité scientifique de la revue Narcomafie et de la Biblioteca digitale sulla camorra e sulla cultura della legalità [bibliothèque numérique sur la camorra et la culture de la légalité] près l'Université « Federico II" de Naples.

Parmi ses travaux, on peut citer : Napoli... Serenata calibro 9. Storia e immagini della camorra tra cinema sceneggiata e neomelodici (2007) ; Il sindaco gentile. Gli appalti, la camorra e un uomo onesto. La storia di Marcello Torre (2016); La nazione del miracolo. L'Italia e gli italiani tra storia, memoria e immaginario (2018). Ainsi que les anthologies : Strozzateci Tutti (2010), Novantadue. L'anno che cambiò l'Italia (Castelvecchi 2012) et Dialoghi sulle mafie (2015). Il a également participé aux ouvrages collectifs L'Atlante delle mafie (2012 et 2015) et Riformismo mancato. Società, consumi e politica nell'Italia del miracolo (2014). 